\title{
ANÁLISE ESPACIAL DE UM FRAGMENTO FLORESTAL BASEADA NO MOSAICO DE DIRICHLET ${ }^{1}$
}

\author{
João Domingos Scalon², Carlos Alberto Púpio de Oliveira ${ }^{3}$ e José Márcio de Mello ${ }^{4}$
}

\begin{abstract}
RESUMO - A configuração espacial das árvores afeta grande número de processos fisiológicos e ecológicos em uma floresta, incluindo competição, distribuição, tamanho, crescimento e mortalidade da espécie. Métodos baseados na função $K$ de Ripley têm sido usados com frequência para caracterizar a configuração espacial de uma floresta. Neste artigo foram propostos alguns métodos, que são baseados nas áreas do mosaico de Dirichlet (função $D$ ), para descrever a distribuição espacial de árvores. Devido à importância da Xylopia brasiliensis (pindaíba) na estrutura e dinâmica de floresta Semidecidual Montana, este trabalho avaliou as funções $K$ e $D$ para descrever a distribuição espacial da espécie. Os resultados indicaram que os estimadores das funções $K$ e $D$, combinados com simulações Monte Carlo, levaram à rejeição da hipótese de completa aleatoriedade espacial $(\mathrm{p}<0,10)$ da Xylopia brasiliensis em favor da presença de agrupamento espacial da espécie dentro do fragmento florestal.
\end{abstract}

Palavras-chave: Manejo, Método de Monte-Carlo e Mosaico de Voronoi.

\section{SPATIAL ANALYSIS OF A FOREST FRAGMENT BASED ON DIRICHLET'S MOSAIC}

\begin{abstract}
The spatial pattern of trees affects a large number of physiological and ecological processes in a forest, including competition, distribution, size, growth and mortality of the species. Methods based on the Ripley's K function have been frequently used to characterize the spatial configuration of a forest. In this article we advocate to use methods that are based on areas of the Dirichlet's mosaic (function D) to describe the spatial distribution of trees. Due to the importance of Xylopia brasiliensis (Pindaíba) in the structure and dynamics of Semideciduous montana forests, this study assessed the $\mathrm{K}$ and $\mathrm{D}$ functions to describe the spatial distribution of this specie. Results showed that the estimators of the $\mathrm{K}$ and $\mathrm{D}$ functions, combined with Monte Carlo simulations, led to rejection of the null hypothessis of completely spatial randomness ( $p$ $<0,10)$ of the Xylopia brasiliensis in favor of the presence of clustering of the specie within the forest fragment.
\end{abstract}

Keywords: Management, Monte Carlo Method and Voronoi's Mosaic.

\section{INTRODUÇÃO}

A espécie Xylopia brasiliensis, da família Annonaceae, também conhecida por diversos nomes regionais, tal como pindaíba, é uma espécie perenifólia presente em solos de rápida drenagem. A espécie distribui-se desde o Sul da Bahia até o Rio Grande do Sul, geralmente em floresta pluvial da encosta atlântica, sendo também observada nas Florestas de Galeria e Florestas Estacionais Semideciduais da Região Sudeste (JUSTO, 206). A madeira da espécie pode ser empregada na construção civil ou, ainda, no paisagismo e arborização urbana (JUSTO, 2003; LORENZI, 1992). Nos últimos anos, a espécie Xylopia brasiliensis tem despertado

\footnotetext{
${ }^{1}$ Recebido em 14.06.2011 e aceito para publicação em 28.05.2012.

${ }^{2}$ Universidade Federal de Lavras, Departamento de Ciências Exatas. CAMPUS UNIVERSITÁRIODA UFLA. E-mail: <scalon@ dex.ufla.br>.

${ }^{3}$ Universidade Federal do Acre, Campus Floresta em Cruzeiro do Sul. E-mail: carlos.pupio@ gmail.com

${ }^{4}$ Universidade Federal de Lavras, Departamento de Ciências Florestais. CAMPUS UNIVERSITÁRIO DA UFLA. E-mail: $<$ josemarcio@dcf.ufla.br>.
} 
interesse científico em razão de suas potencialidades bioquímicas e econômicas, embora não seja plantada para fins comerciais.

Os estudos sobre a espécie Xylopia brasiliensis ainda são escassos para entender sua resposta fisiológica em hábitat natural ou modificado. A obtenção de informações científicas pode ser útil para fornecer subsídio para sua exploração, manejo e conservação da espécie (JUSTO, 2003; CZANK JR., 206). Nesse sentido, o conhecimento do padrão de distribuição espacial da espécie Xylopia brasiliensis pode fornecer informações sobre a ecologia, subsidiar a definição de estratégias de manejo e, ou, conservação ou, simplesmente, esclarecer a configuração espacial da espécie.

Existem evidências sugerindo que a configuração espacial é de fundamental importância para a dinâmica de comunidades de plantas (MARTINEZ et al., 2010; TILMAN; KAREIVA, 1997). A caracterização da distribuição espacial das espécies que compõem uma floresta nativa também pode ser informação extremamente importante para balizar o manejador florestal na definição de critérios para selecionar as espécies a serem removidas da população (BREUGEL, 1996). O conhecimento desse tema, juntamente com a análise estrutural da floresta, particularmente a densidade relativa, aliada a outras informações como a estrutura balanceada da floresta remanescente, pode viabilizar a elaboração e execução de planos de manejo que tenham compromisso com a sustentabilidade da floresta (SCOLFORO, 1998). Assim, a caracterização do padrão de distribuição espacial pode fornecer informações sobre a ecologia, subsidiar a definição de estratégias de manejo e, ou, conservação e auxiliar em processos de amostragem ou, simplesmente, esclarecer a estrutura espacial de uma espécie (SCOLFORO, 1998; MARTINEZ et al., 2010).

Segundo Diggle (2003), caracterizar a distribuição espacial implica testar a hipótese de completa aleatoriedade espacial (CAE) contra duas hipóteses alternativas, padrão agregado ou padrão uniforme (regular). Na literatura, a maior parte dos autores concorda que a distribuição espacial aleatória é rara em populações de plantas, assim como a distribuição regular, e que a maioria das populações tem algum grau de agregação dos indivíduos no espaço (LEGENDRE; FORTIN, 1989).

Um método popular para analisar e caracterizar a distribuição espacial de florestas homogêneas, isto é, estacionárias e isotrópicas, é a "medida de segunda ordem reduzida", também conhecida como função $K$ de Ripley (1976). A principal vantagem do método é que ele permite a detecção do padrão espacial em diferentes escalas de distâncias simultaneamente. Outra vantagem importante é que a função $K$ é bem conhecida sob a hipótese de CAE, o que permite que o padrão espacial observado seja comparado com modelo teórico sob a hipótese de CAE. Não é surpresa, portanto, que a função $K$ seja extensivamente utilizada na caracterização espacial de florestas, como pode ser observado nos trabalhos de Diggle (2003), Czank Jr. (2006), Silva et al. (2008), Comas et al. (2009) e Martinez et al. (2010), entre outros.

Deve-se observar que a função $K$ de Ripley é restrita apenas para configurações estacionárias. Entretanto, as configurações espaciais de florestas são, raramente, estacionárias. A fertilidade do solo, a presença de um rio ou simplesmente a heterogeneidade do ambiente podem provocar estruturas florestais não homogêneas (COMAS et al., 2009).

Alguns autores (DIGGLE, 2003; MYLES et al., 1995) sugerem que a análise dos mosaicos de Dirichlet (ou Voronoi) pode ser utilizada para caracterizar uma configuração espacial de pontos. O principal objetivo deste artigo é mostrar como o uso das áreas do mosaico de Dirichlet, que não requer a suposição de configuração estacionária, pode ser usado para caracterizar a configuração espacial de florestas. Assim, este artigo propõe métodos de Monte Carlo (análise gráfica e testes de hipóteses), tendo como base as áreas do mosaico de Dirichlet (função $D$ ), para testar a hipótese de CAE em florestas. Os métodos são aplicados e comparados com os métodos baseados na função $K$ de Ripley para caracterizar a estrutura espacial de 507 árvores da espécie Xylopia brasiliensis, conhecida pelo nome regional de "pindaíba", em um Fragmento Florestal Estacional Semidecidual Montana, localizado no Município de Lavras, MG.

\section{MATERIAL E MÉTODOS}

\subsection{Dados da espécie Xylopia brasiliensis}

Nesta seção é feita uma descrição da espécie Xylopia brasiliensis localizada no fragmento florestal e da forma como foram obtidos os dados que foram utilizados neste trabalho.

A coleta dos dados (localização das 507 árvores da espécie Xylopia brasiliensis, da família Annonaceae), também conhecida como Pindaíba, foi realizada na Reserva 
Florestal da UFLA, que se localiza no Campus da Universidade Federal de Lavras (2113'40"'S e 44⒌'50"W GRW), na cidade de Lavras, MG, com altitude variando entre 920 e $940 \mathrm{~m}$ e área de 5,8 ha.

A reserva consiste de um Fragmento de Floresta Estacional Semidecidual Montana que se encontrava em processo de regeneração após uma série de perturbações sofridas no passado, como incêndios e exploração descontrolada. O solo da mata foi classificado como Latossolo Vermelho (OLIVEIRAFILHO et al., 1994). O clima regional é do tipo Cwa, segundo a classificação climática de Köppen, com precipitação média anual de $1.530 \mathrm{~mm}$ e $80 \%$ das chuvas concentradas de outubro a março. A estação seca se estende de abril a setembro (BRASIL, 1992).

Os dados deste trabalho foram coletados entre maio e julho de 2005. Foram obtidas as coordenadas $(x, y)$ de todos os indivíduos que apresentaram diâmetro à altura do peito (DAP) superior ou igual a $5 \mathrm{~cm}$. Para obter as coordenadas, foram utilizadas bússola e trena, bem como denominado um marco $(0,0)$ por parcela, sendo, posteriormente, as coordenadas das árvores convertidas para um marco comum entre elas. Observou-se que na área já existiam parcelas permanentes de 20x20, com vértices demarcados com tubos de PVC, as quais foram delimitadas com barbante, de modo a facilitar a coleta das coordenadas (CZANK JR., 2006). A localização de cada uma das árvores na reserva florestal está apresentada na Figura 1 (A). A partir dessas localizações foram aplicados métodos baseados tanto no mosaico de Dirichlet quanto na função $K$ de Ripley, apresentados a seguir, para descrever a distribuição espacial da espécie Xylopia brasiliensis.

\subsection{A função $D$ de Dirichlet}

Nesta seção são apresentados o mosaico de Dirichlet (ou diagrama de Voronoi), o estimador da função de distribuição das áreas das regiões do mosaico, as estatísticas de teste e o processo de Monte-Carlo envolvido para testar a hipótese de completa aleatoriedade espacial.

Um processo pontual espacial é definido como um conjunto de $n$ eventos $\left\{X_{1}, \ldots . . X_{n}\right\}$ irregularmente distribuído no plano cuja localização $\left(x_{i}, y_{i}\right)$ foi gerada por um mecanismo estocástico (DIGGLE, 2003).
O mosaico de Dirichlet divide a área de amostragem da configuração pontual em regiões (células), de tal forma que a região $D_{i j}$ contém apenas o ponto $X_{i}$. Além disso, todas as regiões vizinhas de $D_{i}$ estão mais próximas de $X_{i}$ do que qualquer outro ponto dentro da área de amostragem. O polígono de Dirichlet refere-se à fronteira da célula de Dirichlet. O mosaico de Dirichlet também é conhecido como diagrama de Voronoi, tesselação de Voronoi, decomposição de Voronoi e tesselação de Dirichlet (AURENHAMMER, 1991).

Os tamanhos das áreas do mosaico fornecem uma ideia do tipo de configuração espacial, conforme pode ser visto na Figura 1 (B). Se as áreas do mosaico forem, aproximadamente, iguais, têm-se evidências de regularidade. Caso algumas regiões do plano apresentem mosaicos com áreas muito pequenas, sendo envolvidas por mosaicos com áreas muito grandes, ou vice-versa, então há indícios de uma configuração que apresenta agrupamentos. Para determinar, estatisticamente, os tamanhos e formas das áreas do mosaico para levar à rejeição da hipótese nula de uma configuração gerada por um processo CAE, calcula-se a área de cada célula do mosaico de Dirichlet. Então, Myles et al. (1995) sugeriram estimar $D(r)$, ou seja, a função distribuição das áreas das regiões do mosaico de Dirichlet, através da equação:

$$
\hat{D}(r)=\frac{U(r)}{n}
$$

em que $n$ é o número de áreas do mosaico, $r$ é uma medida de área e $U(r)$ é o número total de mosaicos com áreas inferiores a $r$.

A função $D$ teórica para um processo de Poisson homogêneo com área infinita não é conhecida. Para contornar esse problema, podem-se gerar uma série de configurações sob a hipótese de CAE e calcular $\hat{D}(r)$ para cada uma das configurações. A seguir, a função $D$, sob a hipótese de CAE, pode ser obtida pelo valor médio do conjunto.

O estimador, combinado com simulação Monte Carlo, pode ser utilizado para testar a hipótese nula de que os eventos seguem uma distribuição de Poisson homogênea, ou seja, que as árvores apresentam CAE na floresta.

Para construção exata do teste contra a hipótese de CAE, primeiramente define-se $u_{i}$ como a medida da discrepância entre a função observada e a função teórica

Revista Árvore, Viçosa-MG, v.36, n.4, p.733-740, 2012 
sob a suposição de CAE sobre toda a área $r$. Diggle (2003) sugeriu que essa medida pode ser obtida tanto pela distância de Cramer-Von Mises quanto pela distância de Kolmogorov dadas, respectivamente, por:

$$
\begin{gathered}
u_{i}=\int_{0}^{r_{0}}\left\{\hat{D}_{i}(r)-D(r)\right\}^{2} d r \\
u_{i}=\max _{0 \leq x \leq r_{0}}\left|\hat{D}_{i}(r)-D(r)\right|
\end{gathered}
$$

para $u_{i}: \mathrm{i}=2, \ldots s$, em que $u_{i}$ é a medida para o processo pontual observado e $d r$ é a diferencial em relação a $r$. Se ao ordenar $u_{i}$ obtiver $u_{i}$ em um dos extremos da distribuição, há vidências estatísticas para se rejeitar a hipótese de completa aleatoriedade espacial. Mais formalmente, se $u_{i}$ é o $k$-ésimo maior valor, então se rejeita a hipótese nula caso $P=(s+1-k) / s$. seja inferior ou igual ao nível de significância $\alpha=0,10$, em que $P$ é o valor- $p$ empírico, $s$ é o número de simulações de Monte Carlo e $k$ é a posição de $u_{i}$.

Os testes propostos anteriormente não indicam a direção (regularidade ou agrupamentos) da rejeição da hipótese nula de CAE. Para tal, pode-se utilizar o seguinte procedimento gráfico descrito em Diggle (2003).

Seja $\hat{D}_{i}(r): i=2,3, \ldots, s$ calculada a partir dos dados de uma configuração pontual observada ea função de distribuição empírica calculada para cada $(s-1)$ simulações independentes de $n$ eventos independentes e identicamente distribuídos na região observada sob a suposição de CAE. Então, define-se o envelope de simulações superior e inferior como:

$$
\begin{gathered}
S(r)=\max \left\{\hat{D}_{i}(r)\right\}, i=1, \ldots, s \\
I(r)=\min \left\{\hat{D}_{i}(r)\right\}, i=1, \ldots, s
\end{gathered}
$$

Os valores de $\hat{D}_{1}(r) S(r)$ e $I(r)$ são colocados no eixo $y$ do gráfico, enquanto as médias das áreas são colocadas no eixo $x$. Se a linha de estiver posicionada acima do envelope, então há evidências de ocorrência de agrupamentos na escala de área considerada. Se a linha $\hat{D}_{1}(r)$ estiver posicionada abaixo do envelope, há evidências estatísticas de regularidade nessa escala de área.

Revista Árvore, Viçosa-MG, v.36, n.4, p.733-740, 2012

\subsection{A função $K$ de Ripley}

Nesta seção é apresentada como a função $K$ de Ripley pode ser utilizada para testar a hipótese de completa aleatoriedade espacial de uma configuração pontual.

A função $K$, proposta por Ripley (1976), é definida como $K(t)=\lambda^{-1} E$ [número de eventos contidos dentro de uma distância $t$ a partir de um evento arbitrário], em que $E$ é o operador esperança e ë é a intensidade, ou número médio de eventos por unidade de área, em uma região assumida como constante. $\mathrm{O}$ mais simples e natural estimador de $K(t)$ que corrige o efeito de bordas é dado por:

$$
\hat{K}(t)=\frac{|A|}{n^{2}} \sum_{i=1}^{n} \sum_{j=1}^{n} \frac{I_{t}\left(u_{i j}\right)}{w_{i j}}
$$

em que é a distância entre os eventos e $j(i \neq j)$, $I_{t}\left(u_{i j}\right)$ é uma função indicadora igual a 1 quando é menor que as distâncias $t$ e 0 ; caso contrário, $n$ é o número de evento no mapa analisado com área $|A|$ e $w_{i j}$ é um fator de correção que representa a proporção da circunferência ao redor de um evento $i$, passando sobre o evento $j$ que está dentro de $|A|$.

A função $K$ teórica para um processo de Poisson homogêneo com área infinita é dada pela equação $\hat{K}_{i}(t)=\pi t^{2}$. Portanto, sob agrupamento, espera-se que $\hat{K}_{i}(t)>\pi t^{2}$ e, no caso de regularidade, $\hat{K}_{i}(t)<\pi t^{2}$. Para facilitar a interpretação da função $K$, é usual utilizar um estimador linear centrado em zero dessa função, denominada função $L$ e definida pela equação $\hat{L}(t)=\sqrt{\frac{\hat{K}(t)}{\pi}}-t$. A partir desse estimador, podem-se utilizar os mesmos procedimentos de Monte Carlo vistos anteriormente (DIGGLE, 2003).

Para obter as estatísticas de testes, foi utilizado $\mathrm{o}$ valor da distância $x_{o}=0,25 \mathrm{x}$ (maior distância entre duas espécies) para a função $K \mathrm{e} r_{o}=0,1$ x ( 5,8 hectares) para a função $D$ como o limite superior tanto na medida de Cramer-von Mises quanto na medida de Kolmogorov. O valor teórico da função $D$, sob a hipótese de CAE, foi calculado a partir de 1.000 simulações, com 507 pontos distribuídos aleatoriamente em um plano com área de 5,8 hectares. Para obter o valor- $p$ dos testes de hipótese, utilizaram-se 100 simulações de Monte Carlo, atendendo 
ao que é recomendado por Diggle (2003). Em todas as estatísticas de testes onde foi utilizada a distância de Cramer-von Mises, a integral foi calculada numericamente usando-se o método do trapézio. Todos os cálculos foram realizados utilizando programas desenvolvidos na linguagem $\mathrm{R}$ (R DEVELOPMENT CORE TEAM, 2011). Esses programas utilizaram várias funções disponíveis na biblioteca spatstat (BADDELY; TURNER, 2005) da plataforma $\mathrm{R}$.

\section{RESULTADOS}

A Figura 1 mostra a posição e o mosaico de Dirichlet dos 507 indivíduos da espécie Xylopia brasiliensis no fragmento florestal. A análise visual da Figura $1 \mathrm{~A}$ não possibilita uma decisão quanto ao tipo de distribuição das árvores de pindaíba, apesar de sugerir a presença de agrupamentos. Na Figura 1B, a presença de agrupamentos é realçada pelos polígonos de Dirichlet.

A Tabela 1 apresenta os resultados dos testes de Monte Carlo, mostrando que estes rejeitam a hipótese de distribuição aleatória das árvores de pindaíba no fragmento florestal a $10 \%$ de significância. Entretanto, a Tabela 1 mostra que a função $K$ apresenta valores$p$ bem menores $(\mathrm{p}=0,01)$ que a função $D(\mathrm{p}=0,10)$, em ambas as medidas.

Os resultados apresentados na Tabela 1 não permitem avaliar a direção da rejeição da hipótese de CAE. Assim, conduziu-se a análise gráfica pelas funções $L$ e $D$ descritas no item 2.2. A Figura 2 mostra as funções $L$ (Figura 2A) e $D$ (Figura 2B) observadas (linha cheia) e os envelopes resultantes de 1.000 simulações (linhas tracejadas) sob a hipótese de CAE. Pode-se observar, na Figura 2A, que a função $L$ detectou agregação em todo o espectro de distância analisada na pindaíba, enquanto a Figura 2B mostra que a função $D$ detectou agregação das árvores de pindaíba até $150 \mathrm{~m}^{2}$ no fragmento florestal.

\section{DISCUSSÕES}

Iniciou-se a análise do padrão da distribuição espacial da espécie Xylopia brasiliensis através de uma análise gráfica exploratória, conforme apresentado na Figura1. Uma análise visual da Figura 1A não possibilita uma decisão quanto ao tipo de distribuição das árvores de pindaíba, apesar de sugerir a presença de agrupamentos. Entretanto, na Figura 1B a presença de agrupamentos é realçada pelos polígonos de Dirichlet, uma vez que o mosaicoé formado por polígonos convexos, e suas áreas são menores em regiões com alta intensidade de pontos e maiores em regiões com baixa intensidade de pontos.

Apesar de a Figura 1B mostrar indícios de presença de agrupamentos de pindaíba no fragmento florestal, é importante caracterizar estatisticamente a significância
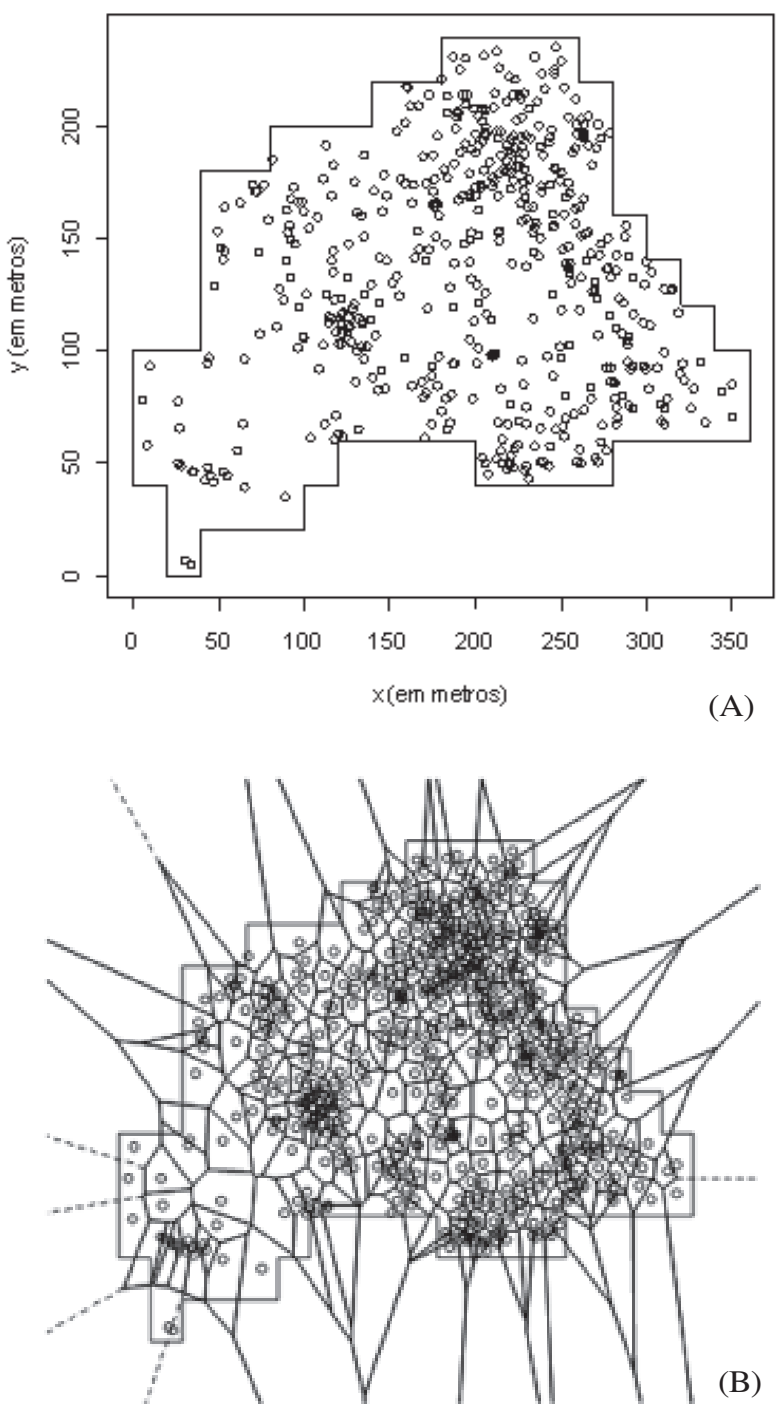

Figura 1 - Localização (A) e o mosaico de Dirichlet (B) dos 507 indivíduos da espécie Xylopia brasiliensis em um Fragmento Florestal Estacional Semidecidual Montana localizado em Lavras, Minas Gerais.

Figure 1 -Locations (A) and the Dirichlet tessellation (B) for the pattern of 507 individuals of Xylopia brasiliensis in a montana semideciduous seasonal forest fragment located in Lavras, Minas Gerais.

Revista Árvore, Viçosa-MG, v.36, n.4, p.733-740, 2012

\section{Siff}


desses indícios. Para tal propósito foram desenvolvidos diversos testes e procedimentos gráficos baseados nas funções $F, G, K$ e $J$. Esses testes estão descritos em detalhes em Diggle (2003). A função $K$ é considerada
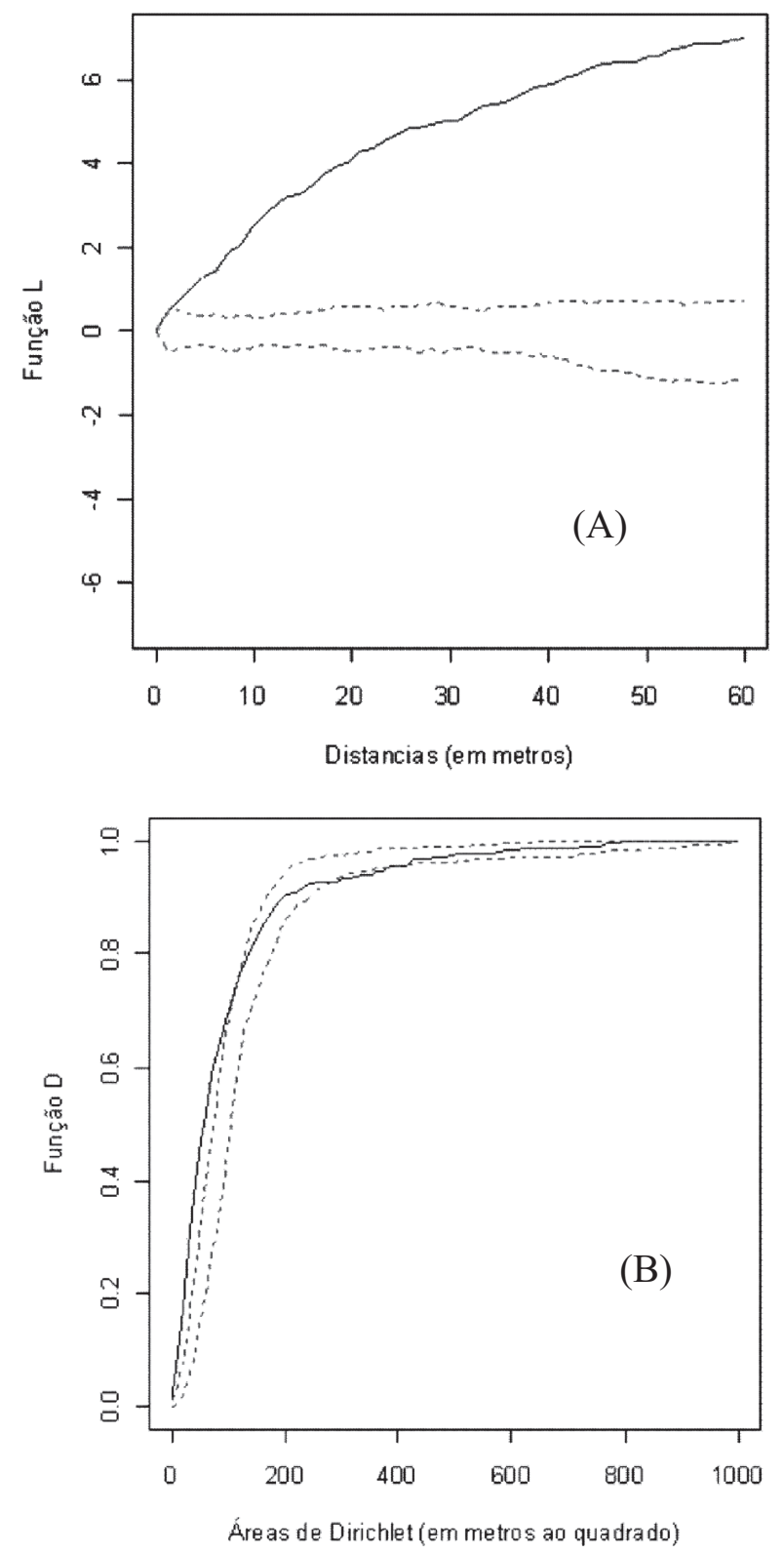

Figura 2 - Funções $L$ (A) e $D$ (B) observadas (linha cheia) e envelope com 1.000 simulações (linhas tracejadas) sob a hipótese de completa aleatoriedade espacial.

Figure 2 -Observed $L(A)$ and $D(B)$ functions (solid line) and envelope of 1000 of completely spatial randomness simulations (dashed lines). uma das funções mais poderosas para detectar configurações com agrupamentos em diferentes escalas e também a mais utilizada em ciências florestais (DIGGLE, 2003; CZANK JR., 2006; MARTINEZ et al., 2010). Assim, essa função foi utilizada como parâmetro de comparação com a função $D$, proposta neste artigo, para caracterizar a estrutura espacial das árvores de pindaíba no fragmento florestal. Assim, foram aplicados os testes de hipóteses descritos no item 2.2, cujos resultados estão apresentados na Tabela 1 .

Os resultados da Tabela 1 mostram claramente que os testes baseados tanto na função $K$ quanto na função $D$ rejeitam a hipótese de CAE das árvores da espécie Xylopia brasiliensis a 10\% de significância. Entretanto, a função $K$ apresenta valores- $p(\mathrm{p}=0,01)$ bem menores que a função $D(\mathrm{p}=0,10)$, em ambas as medidas.

Oliveira (2010) mostrou que em configurações com a presença de agrupamento bem definidos, as funções $K$ e $D$ apresentam testes com o mesmo poder contra a hipótese de CAE. Entretanto, conforme a configuração se aproxima da aleatoriedade espacial, a função $K$ apresenta testes que são mais poderosos que a função $D$. Os resultados obtidos por Oliveira (2010) corroboram os de Myles et al. (1995), ou seja, de que a função $D$ pode apresentar testes contra a hipótese de CAE que, apesar de serem menos poderosos do que os testes baseados na função $K$, podem ser a alternativa para essa função.

Os resultados apresentados na Tabela 1 não permitem avaliar se a rejeição da hipótese de CAE foi em direção de distribuição com agrupamentos ou regularidade das árvores. Assim, conduziu-se a análise gráfica utilizando as funções $L$ e $D$ descritas no item 2.2. O resultado apresentado na Figura 2A para essa função mostra que a linha referente à função observada está acima

Tabela 1 - Estatística de teste (valor-p) contra a hipótese de completa aleatoriedade espacial da espécie Xylopia brasiliensis.

Table 1 -Test statistic ( $p$-value) against the null hypothesis of completely spatial randomness of the specie Xylopia brasiliensis.

\begin{tabular}{lll}
\hline & $\begin{array}{l}\text { Distância de } \\
\text { Kolmogorov }\end{array}$ & $\begin{array}{l}\text { Distância de } \\
\text { Cramer-Von Mises }\end{array}$ \\
\hline Função $D$ & $0,56(p=0,10)$ & $0,55(p=0,10)$ \\
Função $K$ & $7,00(p=0,01)$ & $2789,44(p=0,01)$ \\
\hline
\end{tabular}

Revista Árvore, Viçosa-MG, v.36, n.4, p.733-740, 2012 
do envelope superior em todo o espectro de distância analisada, o que fornece evidências estatísticas a 99\% de confiança de que a hipótese nula de CAE deve ser rejeitada em favor da existência de agrupamentos. Fazendo a mesma análise para a função D (Figura 2B), observa-se que a linha referente à função observada está acima do envelope superior até $150 \mathrm{~m}^{2}$, o que mostra que a função $D$ também detectou agregação das árvores de pindaíba. A Figura 2B também mostra que o gráfico da função $L$ é mais explícito na detecção da não aleatoriedade espacial da espécie. Entretanto, esse fato é devido à escala dos dois gráficos. Enquanto na função $L$ a escala é uma medida de distância, na função $D$ a escala é uma medida de área.

Os resultados deste artigo corroboram as afirmações de Legendre e Fortin (1989) e de outros autores que defendem que a maioria das populações florestais tem algum grau de agregação dos indivíduos no espaço.

Czank Jr. (2006) observou que configurações que exibem padrões agregados em florestas podem indicar clareiras produzidas pela queda de árvores. Essa situação pode ser um dos fatores responsáveis pelos agrupamentos na área de estudo que é, regularmente, afetado por focos de incêndios em períodos de seca.

Outro fator que pode ser responsável pela presença de agrupamentos é o fato de que a área do estudo se trata de pequeno fragmento isolado urbano que não está livre de exploração ilegal. Lorenzi (1992) e Czank Jr. (2006) afirmaram que a madeira da Pindaíba, apesar de baixa durabilidade natural, vem sendo empregada internamente em construção civil, como tabuado, caibros e vigas. A árvore ainda é utilizada na arborização urbana e no paisagismo e, portanto, esse fator não deve ser totalmente descartado.

Além dos fatores mencionados anteriormente, podem existir fatores ambientais como a disponibilidade de recursos e condições microclimáticas que podem também estar atuando sobre a configuração espacial da espécie Xylopia brasiliensis no fragmento florestal (SCOLFORO, 1998; SILVA et al., 2008).

Evidentemente, somente mais estudos científicos e observações coletadas na área de estudo podem fornecer subsídios para o conhecimento dos verdadeiros fatores responsáveis pelos agrupamentos da espécie Xylopia brasiliensis no Fragmento Florestal Estacional Semidecidual Montana.

\section{CONCLUSÕES}

Os resultados deste artigo indicaram que os métodos baseados nas funções $D$ e $K$ levaram às mesmas conclusões quanto à configuração espacial das árvores da espécie Xylopia brasiliensis, apesar de a função $K$ apresentar, nos testes de hipóteses, valores- $p$ menores que a função $D$. Com base nos resultados, pode-se concluir que as árvores desta espécie se distribuem de forma agregada no fragmento florestal. Os resultados também indicaram que os testes baseados na função $D$ podem ser utilizados como forma alternativa, ou complementar, aos testes baseados na função $K$ de Ripley para testar a hipótese de completa aleatoriedade espacial de espécies florestais, principalmente quando houver indícios de configurações não estacionárias.

\section{AGRADECIMENTOS}

Aos três revisores, pelas sugestões que contribuíram para a melhoria deste trabalho.

\section{REFERÊNCIAS}

AURENHAMMER, V. Voronoi diagrams: a survey of a fundamental geometric data structure. ACM Computing Surveys, v.23, n.3, p.345-405, 1991.

BADDELEY, A.; TURNER, R. Spatstat: An R package for analyzing spatial point patterns. Journal of Statistical Software, v.12, n.6, p.1-42, 2005.

BRASIL. Ministério da Agricultura e Reforma Agrária. Normais Climatológicas de 1961-1990. Brasília: SNI/DNM, 1992. 84p.

BREUGEL, V. P. Spatial distribution of three commercial tree species in a neotropical rain forest. Wageningen: Agricultural University, 1996. 107p.

COMAS, C. et al. Characterising forest spatial structure through inhomogeneous second order characteristics. Stochastic Environmental Research and Risk Assessment, v.23, n.3, p.387-397, 2009.

Revista Árvore, Viçosa-MG, v.36, n.4, p.733-740, 2012 
CZANK JR., L. Análise da distribuição espacial de indivíduos de xylopia brasiliensis em um fragmento de floresta estacional semidecidual Montana em Lavra-MG. 2006. 33f. Monografia (Bacharelado em Engenharia Florestal). Universidade Federal de Lavras, Lavras, 2006.

DIGGLE, P. J. Statistical analysis of spatial point patterns. London: Academic Press, 2003. 272p.

JUSTO, C. F. Aspectos ecofisiológicos de plantas jovens de Xylopia brasiliensis Sprengel em sub-bosque de mata e de eucaliptal. Lavras: Universidade Federal de Lavras, 2003. 117p.

LEGENDRE, P.; FORTIN, M. Spatial pattern and ecological analysis. Vegetatio, v.80, p.107-138, 1989.

LORENZI, H. Árvores brasileiras: manual de identificação e cultivo de plantas arbóreas nativas do Brasil. Nova Odessa: Plantarum, 1992. 352p.

MARTINÉZ, I. et al. Spatial associations among tree species in a temperate forest community in North-western Spain. Forest Ecology and Management, v.260, n.4, p.456-465, 2010.

MYLES, J. P. et al. Statistical tests for clustering of second phases in composite materials.

Philosophical Magazine A, v.72, n.2, p.515-528, 1995.
OLIVEIRA, C. A. P. O uso da função de Dirichlet em testes de hipótese da aleatoriedade espacial em configurações pontuais. 2010. $81 \mathrm{f}$. Dissertação (Mestrado em Estatística e Experimentação Agropecuária)) - Universidade Federal de Lavras. Lavras, 2010.

OLIVEIRA FILHO, A. T. et al. Composição florística e estrutura comunitária de um remanescente de floresta semidecídua montana em Lavras, MG. Revista Brasileira de Botânica, v.17, n.2, p.167-182, 1994.

R DEVELOPMENT CORE TEAM. R: a language and environment for statistical computing. Viena: R Foundation for Statistical Computing. Disponível em: <http:// www.R-project.org > 2011

RIPLEY, B. D. The second-order analysis of stationary point processes. Journal of Applied Probability, v.13, n.2, p.255-266, 1976.

SCOLFORO, J. R. S. Manejo florestal. Lavras: Universidade Federal de Lavras, 1998. 438p.

SILVA, M. A. et al. Análise da distribuição espacial da candeia (Eremanthus erytropappus (DC.) MacLeish) sujeita ao sistema de manejo porta-semente. Cerne, v.14, n.4, p.311-316, 2008.

TILMAN, D.; KAREIVA, P. M. Spatial ecology: the role of space in population dynamic and interspecific interactions. Princeton: Princeton University Press, 1997. 416p. 